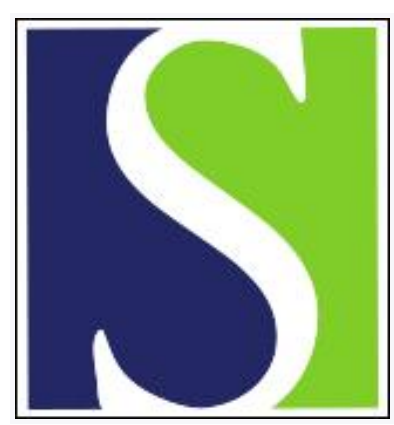

Scand J Work Environ Health 1992;18(5):302-309

https://doi.org/10.5271/sjweh.1572

Issue date: 01 Oct 1992

Menstrual-cycle characteristics and work conditions of workers in poultry slaughterhouses and canneries.

by Messing K, Saurel-Cubizolles MJ, Bourgine M, Kaminski M

Affiliation: CINBIOSE, Universite du Quebec a Montreal, Quebec, Canada.

The following article refers to this text: 2015;41(2):194-203

This article in PubMed: www.ncbi.nlm.nih.gov/pubmed/1439657

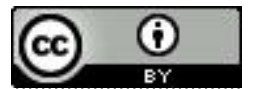




\title{
Menstrual-cycle characteristics and work conditions of workers in poultry slaughterhouses and canneries
}

\author{
by Karen Messing, PhD, ${ }^{1}$ Marie-Josèphe Saurel-Cubizolles, $\mathrm{PhD},{ }^{2}$ Madeleine Bourgine, ${ }^{2}$ \\ Monique Kaminski
}

\begin{abstract}
MESSING K, SAUREL-CUBIZOLLES M-J, BOURGINE M, KAMINSKI M. Menstrual-cycle characteristics and work conditions of workers in poultry slaughterhouses and canneries. Scand $J$ Work Environ Health 1992;18:302 - 9. The food and agriculture industry employs $14.6 \%$ of the female industrial work force in France. Workers are exposed to a variety of environmental and organizational constraints (eg, irregular schedules, cold, uncomfortable postures, repetitive movements). In 1987-1988 a medical examination and questionnaire were administered to 726 workers with menstrual periods in 17 poultry slaughterhouses and six canning factories. Anomalies (irregular cycles, amenorrhea, long cycles) during the previous year were associated with work conditions. After adjustment for relevant nonoccupational variables, irregular cycles were significantly related to schedule variability and cold exposure, amenorrhea was associated with cold exposure, and long cycles with schedule variability. Other parameters such as repetitive work, standing posture, lifting weights, job satisfaction, and hours of domestic work were not associated with cycle anomalies. Cycle anomalies may be a useful indicator of occupational effects on female reproduction, analogous to the use of sperm parameters to warn of effects on male workers.
\end{abstract}

Key terms: amenorrhea, cold exposure, epidemiologic study, menstrual disorders, nonoccupational factors, occupation, repetitive work, tobacco use.

The food and agriculture industry employs $14.6 \%$ of the female industrial work force in France (1). Because of variations in supply and demand, many of these workers have very irregular schedules. Hours of starting and ending work may vary from day to day in an unpredictable way, as does the length of the workday and even the work week. There are peak periods which may last several weeks during which hours may be even more irregular. This work environment also involves other stressors. Temperatures can be cold, especially in slaughterhouses, where temperatures in some areas may go below freezing. Many workers do their jobs standing without moving, on assembly lines, doing repetitive manipulations $(2,3)$.

Although it has been shown that men and women assigned to the same tasks in this industry report similar symptom profiles (4), female workers in poultry slaughterhouses are exposed to some risk factors different from those of men. They tend to be concentrated in certain tasks, most often toward the middle of the processing operation (2). Many more women were as-

\footnotetext{
1 Centre pour l'étude des interactons biologiques entre la santé et l'environnement (Center for the Study of Biological Interactions Between Health and the Environment), Université du Québec à Montréal, Québec, Canada.

2 Institut National de la Santé et de la Recherche Médicale (INSERM) (National Institute of Health and Medical Research), Unit 149, Villjejuif, France.
}

Reprint requests to: Dr K Messing, CINBIOSE, Université du Québec à Montréal, CP 8888, Succursale A, Montréal, Québec $\mathrm{H} 3^{\circ} \mathrm{C} 3 \mathrm{P} 8$, Canada. signed to eviscerating, weighing, cooking, and preparing chickens. While more men lift heavy weights, women more often do repetitive, monotonous tasks, work on the assembly line, and are exposed to cold. In addition the irregularly paid work schedules of most women must be juggled with domestic responsibilities, which add many hours to the work week.

In the context of the current interest in the effects of employment on reproduction $(5,6)$, the effects of work in this industry on parameters involved with the menstrual cycle should be examined. These parameters may constitute an indicator of the effects of work conditions on the reproductive health of women. Disorders of the menstrual cycle have been explored in relation to occupational exposures to synthetic hormones $(7,8)$, organic solvents $(9)$, carbon disulfide $(10)$, and night work (11), although confounding factors were not examined in these studies. Amenorrhea has been associated with strenuous jobs, such as being an athlete (12) or ballet dancer (13), but not with styrene exposure (14). No occupational variables have been considered in relation to cycle length, although exercise has been associated with long cycles among college students (15). Confounding factors have not been controlled for in previous studies of nonchemical exposures and cycle anomalies.

In 1987-1988 a medical examination and questionnaire were administered to French workers in poultry slaughterhouses and canning factories. The present pa- 
per reports associations between cycle anomalies and various work conditions among women in this group.

\section{Subjects and methods}

\section{Study population}

The study was carried out in $1987-1988$ in 17 poultry slaughterhouses and six canning factories in western France. The places of work were selected so as to include a diversity of subregions and factory sizes. The study population included all workers employed on 1 January 1987, administrative staff excluded. In factories employing fewer than 200 workers, all such employees were included in the sample. In the larger factories, a sample of one-half or one-third (in the largest factories) was randomly selected. This procedure yielded 1686 workers. There were 993 women, of whom 112 were not investigated due to sick leave (19 women), maternity or parental leave ( 51 women), lack of time of the physician ( 5 women), resignation (17 women), firing ( 2 women), retirement ( 3 women), refusal to participate (3 women), or other or unknown reasons (12 known). The remaining 881 women were interviewed and examined. Of these, 87 were eliminated because they reported having reached menopause, 62 because they were currently pregnant or reported having been pregnant during the current year, two because they had had hysterectomies, and four because information was not available on one or more of the parameters under study. Data on menstrual cycle were available for 726 women who had menstrual periods.

Women taking oral contraceptives constituted $34 \%$ of the sample. Their cycles were, as expected, much more regular than those of the other women. They were therefore excluded from the sample, the result being a population skewed towards older workers who were somewhat heavier, whose blood pressure was higher, and who smoked less. (See table 1.)

\section{Instruments used for the data collection}

Data were collected at the annual consultation with the occupational health physician (mandatory in France). The employees filled out a self-administered questionnaire on work conditions, social and demographic

Table 1. Population characteristics according to use of oral contraceptives.

\begin{tabular}{|c|c|c|c|c|c|}
\hline \multirow{2}{*}{ Characteristic } & \multicolumn{2}{|c|}{$\begin{array}{l}\text { Women not taking } \\
\text { oral contraceptives }\end{array}$} & & \multicolumn{2}{|c|}{$\begin{array}{l}\text { Women taking oral } \\
\text { contraceptives }\end{array}$} \\
\hline & $N$ & $\%$ & & $N$ & $\%$ \\
\hline \multicolumn{6}{|l|}{ Age } \\
\hline $\begin{array}{l}<25 \text { years } \\
25-39 \text { years } \\
\geq 40 \text { years } \\
\text { P-value }^{\mathrm{a}}\end{array}$ & $\begin{array}{r}37 \\
321 \\
115\end{array}$ & $\begin{array}{r}7.8 \\
67.9 \\
24.3\end{array}$ & $<0.001$ & $\begin{array}{r}42 \\
191 \\
16\end{array}$ & $\begin{array}{r}16.9 \\
76.7 \\
6.4\end{array}$ \\
\hline \multicolumn{6}{|l|}{ Type of factory } \\
\hline $\begin{array}{l}\text { Slaughterhouse } \\
\text { Cannery } \\
\text { P-value }\end{array}$ & $\begin{array}{l}347 \\
128\end{array}$ & $\begin{array}{l}73.0 \\
27.0\end{array}$ & NS & $\begin{array}{r}179 \\
70\end{array}$ & $\begin{array}{l}71.9 \\
28.1\end{array}$ \\
\hline \multicolumn{6}{|l|}{ Size of factory } \\
\hline $\begin{array}{l}<50 \text { employees } \\
50-199 \text { employees } \\
\geq 200 \text { employees } \\
\mathrm{P}_{\text {-value }}^{\mathrm{a}}\end{array}$ & $\begin{array}{r}28 \\
237 \\
210\end{array}$ & $\begin{array}{r}5.9 \\
49.9 \\
44.2\end{array}$ & NS & $\begin{array}{r}17 \\
116 \\
116\end{array}$ & $\begin{array}{r}6.8 \\
46.6 \\
46.6\end{array}$ \\
\hline \multicolumn{6}{|l|}{ Parity } \\
\hline $\begin{array}{l}\text { No children } \\
1-2 \text { children } \\
\geq 3 \text { children } \\
\text { P-value }\end{array}$ & $\begin{array}{l}123 \\
248 \\
103\end{array}$ & $\begin{array}{l}26.0 \\
52.3 \\
21.7\end{array}$ & NS & $\begin{array}{r}52 \\
142 \\
55\end{array}$ & $\begin{array}{l}20.9 \\
57.0 \\
22.1\end{array}$ \\
\hline \multicolumn{6}{|l|}{$\begin{array}{l}\text { Body mass index } \\
\text { (weight } \cdot \text { height }{ }^{-2} \text { ) }\end{array}$} \\
\hline $\begin{array}{l}\leq 10 \text { th percentile } \\
11-89 \text { th percentile } \\
\geq 90 \text { th percentile } \\
\text { P-value }^{\alpha}\end{array}$ & $\begin{array}{r}47 \\
367 \\
57\end{array}$ & $\begin{array}{l}10.0 \\
77.9 \\
12.1\end{array}$ & 0.070 & $\begin{array}{r}20 \\
211 \\
18\end{array}$ & $\begin{array}{r}8.0 \\
84.7 \\
7.2\end{array}$ \\
\hline \multicolumn{6}{|l|}{ Diastolic blood pressure ${ }^{b}$} \\
\hline $\begin{array}{l}<60 \mathrm{~mm} \mathrm{Hg} \\
60-89 \mathrm{~mm} \mathrm{Hg} \\
\geq 90 \mathrm{~mm} \mathrm{Hg} \\
\text { P-value }\end{array}$ & $\begin{array}{r}229 \\
186 \\
54\end{array}$ & $\begin{array}{l}48.8 \\
39.7 \\
11.5\end{array}$ & $<0.001$ & $\begin{array}{r}158 \\
69 \\
14\end{array}$ & $\begin{array}{r}65.6 \\
28.6 \\
5.8\end{array}$ \\
\hline \multicolumn{6}{|l|}{ Tobacco consumption } \\
\hline $\begin{array}{l}\text { Nonsmoker or ex-smoker } \\
\uparrow-9 \text { cigarettes }(d \\
\geq 10 \text { cigarettes/d } \\
\text { P-value }\end{array}$ & $\begin{array}{r}376 \\
64 \\
34\end{array}$ & $\begin{array}{r}79.3 \\
13.5 \\
7.2\end{array}$ & $<0.001$ & $\begin{array}{r}158 \\
58 \\
33\end{array}$ & $\begin{array}{l}63.4 \\
23.3 \\
13.2\end{array}$ \\
\hline
\end{tabular}

a Chi-square test for association between oral contraceptive use and characteristic.

b $1 \mathrm{~mm} H \mathrm{Hg} \approx 133.322 \mathrm{~Pa}$. 
characteristics, and living conditions. The responses to this questionnaire were gone over with the physician. Further details on work conditions were obtained by interview, and a medical questionnaire covering symptoms, health status, and use of health services was completed by the physician.

The medical questionnaire included a list of questions on the menstrual cycle and contraceptive use. $\mathrm{Cy}$ cle irregularity was scored as positive if the woman reported having irregular cycles during the year prior to the examination, with a difference of at least $7 \mathrm{~d}$ between the length of the shortest and longest cycles. Amenorrhea was scored as positive if the women answered yes to the question "During the past year have you had a period of several months during which you did not menstruate (pregnancy excluded)?'” Cycle length was rated as long if the woman reported a regular cycle of more than $32 \mathrm{~d}$ or an irregular cycle with the average of the lengths of the shortest and longest cycles greater than $32 \mathrm{~d}$. Short cycles $(<23 \mathrm{~d})$ were not related to any work conditions (or confounding factors), and the 17 workers reporting short cycles were therefore added to those with normal cycles in the analysis of long cycles. These lengths were chosen because they represented two standard deviations around the mean of $28 \mathrm{~d}$ found for those with regular cycles.

Potential risk factors considered for this study included both nonoccupational characteristics of the employees and characteristics of their work conditions. Nonoccupational characteristics included the following personal characteristics for which an association with cycle irregularity or infertility has been established or suspected (14-17): contraceptive use (oral contraceptives or intrauterine device or other), age $(<25$, $25-39$, and $\geq 40$ years), body mass index, defined as weight divided by height squared ( $\leq 10$ th percentile, 11-89th percentiles, and $\geq 90$ th percentile), diastolic blood pressure [ $\leq 60,61-89$, and $\geq 90 \mathrm{~mm} \mathrm{Hg}$ $(1 \mathrm{~mm} \mathrm{Hg} \approx 133.322 \mathrm{~Pa}$ )], tobacco use (nonsmoker or ex-smoker, $<10$ cigarettes a day, $\geq 10$ cigarettes a day), parity (nulliparous or $\geq 1$ births).

The work conditions analyzed covered a large number of questions on scheduling. Workers were asked whether they always began (or ended) work at the same time, their usual hour of beginning (ending) work, and

Table 2. Prevalence of cycle anomalies among the final study population.

\begin{tabular}{lrr}
\hline Anomaly & $\mathrm{N}$ & $\%$ \\
\hline Irregular cycles & & \\
$\quad$ No & 404 & 85.1 \\
$\quad$ Yes & 71 & 14.9 \\
Amenorrhea & & \\
$\quad$ No & 415 & 90.0 \\
Yes & 46 & 10.0 \\
Long cycle & & \\
$\geq 33$ d & 430 & 93.9 \\
$<33$ d & 28 & 6.1 \\
\hline
\end{tabular}

their earliest and latest hour of beginning (ending) work. In addition a variety of questions on the following aspects of work were included (yes/no unless otherwise specified): repetitive work, cold exposure, temperatures varying or not varying during the day and over the year, assembly-line work, difficulties in keeping up with the pace of the assembly line, heavy lifting, standing position, moving in place, general job satisfaction, length of domestic work week (hours). The domestic work week was analyzed as a dichotomous variable ( $<20$ versus $\geq 20$ hours a week). This break point split the population in half, as in a previous study, in which domestic work requiring more than $20 \mathrm{~h}$ a week was associated with health problems among full-time hospital workers (18). The reference period for all of the questions was the year preceding the interview.

\section{Data analysis}

In the first stage, cycle characteristics were studied in relation to each of the nonoccupational and occupational characteristics, with the use of the chi-square test or Fisher's exact test, as appropriate. Since the size of the sample did not permit all of the work conditions to be entered into the logistic regression, in a second stage, occupational characteristics associated at $\mathrm{P}<0.1$ with cycle anomalies were grouped into the two classes of schedule variability and temperature exposures. (No other work conditions were associated with cycle anomalies.) Multiple logistic regressions were carried out to estimate the role of representative members of each class of work condition, nonoccupational characteristics which had been related in the crude analysis to at least one indicator of menstrual disorders being controlled for. The numbers varied slightly according to the characteristics because of missing data. Logistic regressions were done for all of the subjects for whom all of the variables included in the model were available.

The tables show the adjusted odds ratios and $95 \%$ confidence intervals. It should be noted that the odds ratios roughly approximate the relative risk in our study when the they are around two, even though the prevalences of cycle anomalies were high. The analysis was carried out with SAS (statistical analysis system) and BMDP (biomedical data package) software at the INSERM computer center, "SC5."

\section{Results}

Table 2 shows the menstrual cycle characteristics for the final study population of women with menstrual periods. The characteristics irregular cycles, amenorrhea, and long cycles cannot be regarded as independent: $58 \%$ of amenorrheics and $84 \%$ of those with long 
cycles described themselves as having irregular cycles, and $51 \%$ of the amenorrheics reported long cycles.

\section{Menstrual cycle and nonoccupational characteristics}

Tobacco use appeared to be associated with irregular cycles and long cycles, but not with amenorrhea (table 3). The relationship between tobacco use and irregular or long cycles, remained significant at $\mathrm{P}<0.10$ after adjustment for the body mass index. Use of an intrauterine device was associated negatively with irregular cycles and amenorrhea. Nulliparity and age of less than 25 years or greater than $\mathbf{4 0}$ years were associated with amenorrhea. It should be noted that these associations may be complex since anomalous cycle characteristics can be associated with difficulties in having children, which may lead to the abandoning of contraception.

\section{Menstrual cycle and occupational characteristics}

Slaughterhouse versus cannery. Irregular cycles and amenorrhea were more prevalent among the slaugh- terhouse workers (17 and $12 \%$, respectively) than among the cannery workers $[10(\mathrm{P}=0.06)$ and 5 $(\mathrm{P}<0.02) \%$, respectively]. It should be noted that $96 \%$ of the slaughterhouse workers, but only $57 \%$ of the cannery workers consistently worked at temperatures below $18^{\circ} \mathrm{C}$. However, variable temperatures were more prevalent in the canneries. The cannery workers' schedules were more regular than the slaughterhouse workers' for all of the measures used in the study.

Schedule variability. One-third of the workers had days when their work began at irregular times, over half had days when their work ended at unpredictable times (table 4), and the vast majority did not know their schedule for the day when they arrived at work (not shown). Even outside of the seasonal peak periods, work weeks were irregular for $30 \%$ of the workers.

Overall, irregular cycles, periods of amenorrhea, and long cycles were associated with most schedule variability (table 4). One exception should be noted. Regular cycles were found more frequently among those

Table 3. Prevalence of cycle anomalies by nonoccupational characteristics of the final study population.

\begin{tabular}{|c|c|c|c|c|c|c|}
\hline \multirow{2}{*}{ Characteristic } & \multicolumn{2}{|c|}{ Irregular cycles } & \multicolumn{2}{|c|}{ Amenorrhea } & \multicolumn{2}{|c|}{ Cycles $\geq 33 d$} \\
\hline & $N$ & $\%$ & $\mathrm{~N}$ & $\%$ & $\mathrm{~N}$ & $\%$ \\
\hline \multicolumn{7}{|l|}{ Age } \\
\hline $\begin{array}{l}<25 \text { years } \\
25-39 \text { years } \\
\geq 40 \text { years } \\
\text { P-value }\end{array}$ & $\begin{array}{r}9 \\
42 \\
18\end{array}$ & $\begin{array}{r}24.3 \\
13.0 \\
15.8 \\
\text { NS }\end{array}$ & $\begin{array}{r}6 \\
22 \\
17\end{array}$ & $\begin{array}{r}16.2 \\
7.1 \\
15.2 \\
0.018\end{array}$ & $\begin{array}{r}13 \\
18 \\
7\end{array}$ & $\begin{array}{l}8.3 \\
5.8 \\
6.3 \\
\text { NS }\end{array}$ \\
\hline \multicolumn{7}{|l|}{ Living partner } \\
\hline $\begin{array}{l}\text { Yes } \\
\text { No } \\
\text { P-value }\end{array}$ & $\begin{array}{l}54 \\
14\end{array}$ & $\begin{array}{r}15.0 \\
13.6 \\
\text { NS }\end{array}$ & $\begin{array}{l}33 \\
11\end{array}$ & $\begin{array}{r}9.4 \\
11.0 \\
\text { NS }\end{array}$ & $\begin{array}{r}24 \\
4\end{array}$ & $\begin{array}{l}6.8 \\
4.1 \\
\text { NS }\end{array}$ \\
\hline \multicolumn{7}{|l|}{ Parity } \\
\hline $\begin{array}{l}\text { No children } \\
\text { One or more children } \\
\text { P-value }\end{array}$ & $\begin{array}{l}18 \\
53\end{array}$ & $\begin{array}{r}14.8 \\
15.1 \\
\text { NS }\end{array}$ & $\begin{array}{l}20 \\
25\end{array}$ & $\begin{array}{r}16.5 \\
7.4 \\
0.004\end{array}$ & $\begin{array}{r}8 \\
19\end{array}$ & $\begin{array}{l}6.8 \\
5.5 \\
\text { NS }\end{array}$ \\
\hline \multicolumn{7}{|l|}{ Intrauterine device } \\
\hline $\begin{array}{l}\text { Yes } \\
\text { No } \\
\text { P-value }\end{array}$ & $\begin{array}{r}9 \\
61\end{array}$ & $\begin{array}{r}8.8 \\
16.4 \\
0.055\end{array}$ & $\begin{array}{r}3 \\
43\end{array}$ & $\begin{array}{r}3.0 \\
11.9 \\
0.009\end{array}$ & $\begin{array}{r}5 \\
23\end{array}$ & $\begin{array}{l}5.0 \\
6.4 \\
\text { NS }\end{array}$ \\
\hline \multicolumn{7}{|l|}{$\begin{array}{l}\text { Body mass index } \\
\text { (weight } \cdot \text { height }{ }^{-2} \text { ) }\end{array}$} \\
\hline $\begin{array}{l}\leq 10 \text { th percentile } \\
11-89 \text { th percentile } \\
>90 \text { th percentile } \\
\text { P.value }^{a}\end{array}$ & $\begin{array}{r}11 \\
54 \\
6\end{array}$ & $\begin{array}{r}22.9 \\
14.7 \\
10.7 \\
\text { NS }\end{array}$ & $\begin{array}{r}2 \\
35 \\
9\end{array}$ & $\begin{array}{r}4.4 \\
9.9 \\
16.1 \\
\text { NS }\end{array}$ & $\begin{array}{r}2 \\
25 \\
1\end{array}$ & $\begin{array}{l}4.6 \\
6.9 \\
1.8 \\
\text { NS }\end{array}$ \\
\hline \multicolumn{7}{|l|}{ Diastolic blood pressure ${ }^{b}$} \\
\hline $\begin{array}{l}<60 \mathrm{~mm} \mathrm{Hg} \\
60-89 \mathrm{~mm} \mathrm{Hg} \\
\geq 90 \mathrm{~mm} \mathrm{Hg} \\
\text { P-value }\end{array}$ & $\begin{array}{r}32 \\
30 \\
8\end{array}$ & $\begin{array}{r}13.8 \\
16.3 \\
14.8 \\
\text { NS }\end{array}$ & $\begin{array}{r}16 \\
22 \\
6\end{array}$ & $\begin{array}{r}7.2 \\
12.2 \\
11.3 \\
\text { NS }\end{array}$ & $\begin{array}{r}13 \\
13 \\
1\end{array}$ & $\begin{array}{l}5.7 \\
7.4 \\
1.9 \\
\text { NS }\end{array}$ \\
\hline \multicolumn{7}{|l|}{ Tobacco consumption } \\
\hline $\begin{array}{l}\text { Nonsmoker or ex-smoker } \\
1-9 \text { cigarettes } / d \\
\geq 10 \text { cigarettes } / d \\
\text { P-value }\end{array}$ & $\begin{array}{r}52 \\
9 \\
10\end{array}$ & $\begin{array}{r}13.8 \\
13.8 \\
29.4 \\
0.049\end{array}$ & $\begin{array}{r}39 \\
4 \\
3\end{array}$ & $\begin{array}{r}10.7 \\
6.4 \\
8.8 \\
\text { NS }\end{array}$ & $\begin{array}{r}20 \\
3 \\
5\end{array}$ & $\begin{array}{r}5.5 \\
4.8 \\
14.7 \\
0.088\end{array}$ \\
\hline
\end{tabular}

a Chi-square test for association between cycle anomaly and individual schedule characteristic.

b $1 \mathrm{~mm} \mathrm{Hg} \approx 133.322 \mathrm{~Pa}$. 
Scand J Work Environ Health 1992, vol 18, no 5

Table 4. Prevalence of cycle anomalies and schedule variability among the final study population. (OR= odds ratio, $95 \% \mathrm{Cl}=95 \%$ confidence intervall)

\begin{tabular}{|c|c|c|c|c|c|c|c|c|c|c|c|c|c|}
\hline \multirow{2}{*}{$\begin{array}{l}\text { Type of } \\
\text { schedule } \\
\text { variability }\end{array}$} & \multirow{2}{*}{$\begin{array}{l}\text { Overall } \\
\text { percent }\end{array}$} & \multicolumn{4}{|c|}{ Irregular cycles } & \multicolumn{4}{|c|}{ Amenorrhea } & \multicolumn{4}{|c|}{ Cycles $\geq 33 d$} \\
\hline & & $\mathrm{N}$ & $\%$ & OR & $95 \% \mathrm{Cl}$ & $N$ & $\%$ & OR & $95 \% \mathrm{Cl}$ & $\mathrm{N}$ & $\%$ & OR & $95 \% \mathrm{Cl}$ \\
\hline Variable beginning & & & & 1.9 & $1.2-3.3$ & & & 1.8 & $1.0-3.4$ & & & 2.2 & $1.0-4.7$ \\
\hline $\begin{array}{l}\text { Yes } \\
\text { No }\end{array}$ & $\begin{array}{l}30 \\
70\end{array}$ & $\begin{array}{l}30 \\
41\end{array}$ & $\begin{array}{l}21.4 \\
12.3\end{array}$ & & & $\begin{array}{l}19 \\
27\end{array}$ & $\begin{array}{r}14.2 \\
8.3\end{array}$ & & & $\begin{array}{l}13 \\
15\end{array}$ & $\begin{array}{l}9.6 \\
4.6\end{array}$ & & \\
\hline $\begin{array}{l}\text { Variable ending } \\
\text { Yes } \\
\text { No }\end{array}$ & $\begin{array}{l}62 \\
38\end{array}$ & $\begin{array}{l}53 \\
18\end{array}$ & $\begin{array}{l}18.1 \\
10.0\end{array}$ & 2.0 & $1.1-3.5$ & $\begin{array}{l}33 \\
13\end{array}$ & $\begin{array}{r}11.6 \\
7.4\end{array}$ & 1.6 & $0.8-3.2$ & $\begin{array}{r}24 \\
4\end{array}$ & $\begin{array}{l}8.4 \\
2.3\end{array}$ & 3.9 & $1.3-11.4$ \\
\hline Variable hours/week ${ }^{a}$ & & & & 1.7 & $1.0-2.8$ & & & 1.8 & $0.9-3.3$ & & & 2.6 & $1.2-5.6$ \\
\hline $\begin{array}{l}\text { Yes } \\
\text { No }\end{array}$ & $\begin{array}{l}30 \\
70\end{array}$ & $\begin{array}{l}28 \\
42\end{array}$ & $\begin{array}{l}19.6 \\
12.7\end{array}$ & & & $\begin{array}{l}19 \\
26\end{array}$ & $\begin{array}{r}13.6 \\
8.2\end{array}$ & & & $\begin{array}{l}14 \\
13\end{array}$ & $\begin{array}{l}9.9 \\
4.1\end{array}$ & & \\
\hline Two shifts & & & & 0.4 & $0.2-1.1$ & & & 0.5 & $0.2-1.5$ & & & 0.7 & $0.2-2.4$ \\
\hline $\begin{array}{l}\text { Yes } \\
\text { No }\end{array}$ & $\begin{array}{l}14 \\
86\end{array}$ & $\begin{array}{r}5 \\
66\end{array}$ & $\begin{array}{r}7.5 \\
16.2\end{array}$ & & & $\begin{array}{r}4 \\
42\end{array}$ & $\begin{array}{r}6.0 \\
10.7\end{array}$ & & & $\begin{array}{r}3 \\
25\end{array}$ & $\begin{array}{l}4.5 \\
6.3\end{array}$ & & \\
\hline
\end{tabular}

a Outside seasonal peak periods.

Table 5. Prevalence of cycle anomalies and microclimate among the final study population. (OR= odds ratio, $95 \% \mathrm{Cl}=95 \%$ confidence interval)

\begin{tabular}{|c|c|c|c|c|c|c|c|c|c|c|c|c|c|}
\hline \multirow{2}{*}{$\begin{array}{l}\text { Microclimate } \\
\text { characteristic }\end{array}$} & \multirow{2}{*}{$\begin{array}{l}\text { Overall } \\
\text { percent }\end{array}$} & \multicolumn{4}{|c|}{ Irregular cycles } & \multicolumn{4}{|c|}{ Amenorrhea } & \multicolumn{4}{|c|}{ Cycles $\geq 33 \mathrm{~d}$} \\
\hline & & $N$ & $\%$ & OR & $95 \% \mathrm{Cl}$ & $\mathbf{N}$ & $\%$ & OR & $95 \% \mathrm{Cl}$ & $\mathrm{N}$ & $\%$ & OR & $95 \% \mathrm{Cl}$ \\
\hline Uncomfortably cold & & & & 1.7 & $1.0-3.0$ & & & 2.1 & $1.1-4.3$ & & & 0.9 & $0.4-2.0$ \\
\hline $\begin{array}{l}\text { Yes } \\
\text { No }\end{array}$ & $\begin{array}{l}57 \\
43\end{array}$ & $\begin{array}{l}47 \\
22\end{array}$ & $\begin{array}{l}17.6 \\
11.1\end{array}$ & & & $\begin{array}{l}32 \\
12\end{array}$ & $\begin{array}{r}12.4 \\
6.2\end{array}$ & & & $\begin{array}{l}14 \\
12\end{array}$ & $\begin{array}{l}5.5 \\
6.1\end{array}$ & & \\
\hline $\begin{array}{l}\text { Reported temperature } \\
<9^{\circ} \mathrm{C} \\
\geq 9^{\circ} \mathrm{C}\end{array}$ & $\begin{array}{l}49 \\
51\end{array}$ & $\begin{array}{l}31 \\
20\end{array}$ & $\begin{array}{l}17.5 \\
11.0\end{array}$ & 1.7 & $0.9-3.1$ & $\begin{array}{l}22 \\
11\end{array}$ & $\begin{array}{r}13.1 \\
6.2\end{array}$ & 2.3 & $1.1-4.9$ & $\begin{array}{r}11 \\
9\end{array}$ & $\begin{array}{l}6.4 \\
5.1\end{array}$ & 1.2 & $0.5-3.3$ \\
\hline $\begin{array}{l}\text { Temperature described } \\
\text { as variable }\end{array}$ & & & & 1.8 & $1.1-3.0$ & & & 1.1 & $0.6-2.0$ & & & 0.8 & $0.4-1.9$ \\
\hline $\begin{array}{l}\text { Yes } \\
\text { No }\end{array}$ & $\begin{array}{l}37 \\
63\end{array}$ & $\begin{array}{l}34 \\
35\end{array}$ & $\begin{array}{l}19.9 \\
12.0\end{array}$ & & & $\begin{array}{l}18 \\
28\end{array}$ & $\begin{array}{l}10.8 \\
10.0\end{array}$ & & & $\begin{array}{r}19 \\
9\end{array}$ & $\begin{array}{l}6.6 \\
5.5\end{array}$ & & \\
\hline
\end{tabular}

Table 6. Prevalence of cycle anomalies and various work characteristics among the final study population. (OR $=$ odds ratio, $95 \% \mathrm{Cl}=95 \%$ confidence interval)

\begin{tabular}{|c|c|c|c|c|c|c|c|c|c|c|c|c|c|}
\hline \multirow{2}{*}{$\begin{array}{l}\text { Work } \\
\text { characteristic }\end{array}$} & \multirow{2}{*}{$\begin{array}{l}\text { Overall } \\
\text { percent }\end{array}$} & \multicolumn{4}{|c|}{ Irregular cycles } & \multicolumn{4}{|c|}{ Amenorrhea } & \multicolumn{4}{|c|}{ Cycies $\geq 33 \mathrm{~d}$} \\
\hline & & $N$ & $\%$ & OR & $95 \% \mathrm{Cl}$ & $N$ & $\%$ & OR & $95 \% \mathrm{Cl}$ & $\mathrm{N}$ & $\%$ & OR & $95 \% \mathrm{Cl}$ \\
\hline Standing position & & & & 0.9 & $0.5-1.6$ & & & 0.9 & $0.5-1.8$ & & & 0.5 & $0.2-1.2$ \\
\hline $\begin{array}{l}\text { Yes } \\
\text { No }\end{array}$ & $\begin{array}{l}69 \\
31\end{array}$ & $\begin{array}{l}47 \\
23\end{array}$ & $\begin{array}{l}14.5 \\
15.5\end{array}$ & & & $\begin{array}{l}31 \\
15\end{array}$ & $\begin{array}{r}9.8 \\
10.6\end{array}$ & & & $\begin{array}{l}15 \\
12\end{array}$ & $\begin{array}{l}4.7 \\
8.4\end{array}$ & & \\
\hline Assembly line & & & & 1.0 & $0.6-1.7$ & & & 0.8 & $0.4-1.6$ & & & 0.7 & $0.3-1.4$ \\
\hline $\begin{array}{l}\text { Yes } \\
\text { No }\end{array}$ & $\begin{array}{l}63 \\
37\end{array}$ & $\begin{array}{l}45 \\
26\end{array}$ & $\begin{array}{l}15.1 \\
15.2\end{array}$ & & & $\begin{array}{l}27 \\
19\end{array}$ & $\begin{array}{r}9.4 \\
11.1\end{array}$ & & & $\begin{array}{l}15 \\
13\end{array}$ & $\begin{array}{l}5.2 \\
7.7\end{array}$ & & \\
\hline Handling weights regularly & & & & 1.5 & $0.6-3.6$ & & & 0.9 & $0.3-2.9$ & & & 1.0 & $0.2-4.4$ \\
\hline $\begin{array}{l}\text { Yes } \\
\text { No }\end{array}$ & $\begin{array}{r}7 \\
93\end{array}$ & $\begin{array}{r}7 \\
64\end{array}$ & $\begin{array}{l}20.6 \\
14.6\end{array}$ & & & $\begin{array}{r}3 \\
43\end{array}$ & $\begin{array}{r}8.8 \\
10.1\end{array}$ & & & $\begin{array}{r}2 \\
26\end{array}$ & $\begin{array}{l}6.1 \\
6.1\end{array}$ & & \\
\hline Overall job satisfaction & & & & 1.2 & $0.7-2.5$ & & & 1.1 & $0.5-2.5$ & & & 1.7 & $0.7-5.0$ \\
\hline $\begin{array}{l}\text { No } \\
\text { Yes }\end{array}$ & $\begin{array}{l}17 \\
83\end{array}$ & $\begin{array}{l}14 \\
57\end{array}$ & $\begin{array}{l}17.7 \\
14.5\end{array}$ & & & $\begin{array}{r}8 \\
36\end{array}$ & $\begin{array}{r}10.4 \\
9.4\end{array}$ & & & $\begin{array}{r}7 \\
21\end{array}$ & $\begin{array}{l}9.1 \\
5.5\end{array}$ & & \\
\hline Household tasks & & & & 1.0 & $0.6-1.8$ & & & 0.9 & $0.5-1.8$ & & & 1.4 & $0.6-3.3$ \\
\hline $\begin{array}{l}\geq 20 \mathrm{~h} / \text { week } \\
<20 \mathrm{~h} / \text { week }\end{array}$ & $\begin{array}{l}50 \\
50\end{array}$ & $\begin{array}{l}30 \\
29\end{array}$ & $\begin{array}{l}15.2 \\
14.7\end{array}$ & & & $\begin{array}{l}19 \\
20\end{array}$ & $\begin{array}{l}10.0 \\
10.4\end{array}$ & & & $\begin{array}{l}14 \\
10\end{array}$ & $\begin{array}{l}7.2 \\
5.2\end{array}$ & & \\
\hline
\end{tabular}

working on a two-shift schedule (changing from the morning to the afternoon shift on a weekly basis) and among those taking meals at irregular times on work- days (not shown). These two work conditions were associated positively with each other and negatively with the other schedule variability characteristics. 
Table 7. Multiple logistic regression models for associations between occupational and nonoccupational variables and menstrual cycle anomalies among the final study population. (95\% Cl $=95 \%$ confidence interval)

\begin{tabular}{|c|c|c|c|c|c|c|}
\hline & \multicolumn{2}{|c|}{ Irregular cycles ${ }^{a}$} & \multicolumn{2}{|c|}{ Amenorrhea ${ }^{2}$} & \multicolumn{2}{|c|}{ Cycles $\geq 33 \mathrm{~d}^{\mathrm{a}}$} \\
\hline & Odds ratio & $95 \% \mathrm{Cl}$ & Odds ratio & $95 \% \mathrm{Cl}$ & Odds ratio & $95 \% \mathrm{Cl}$ \\
\hline $\begin{array}{l}\text { Variable beginning } \\
\text { of workday }\end{array}$ & 2.0 & $1.2-3.6$ & 1.7 & $0.9-3.4$ & 2.4 & $1.0-5.5$ \\
\hline Uncomfortably cold & 1.5 & $0.8-2.6$ & 2.8 & $1.3-5.9$ & 1.0 & $0.4-2.4$ \\
\hline Variable temperature & 1.8 & $1.0-3.2$ & 1.1 & $0.6-2.2$ & 0.9 & $0.4-2.2$ \\
\hline $\begin{array}{l}1-10 \text { cigarettes } / d^{b} \\
>10 \text { cigarettes } / d\end{array}$ & $\begin{array}{l}0.9 \\
2.7\end{array}$ & $\begin{array}{l}0.4-2.2 \\
1.1-6.7\end{array}$ & $\begin{array}{l}0.3 \\
0.7\end{array}$ & $\begin{array}{l}0.1-1.3 \\
0.1-3.3\end{array}$ & $\begin{array}{l}1.1 \\
4.1\end{array}$ & $\begin{array}{l}0.3-4.0 \\
1.3-13.4\end{array}$ \\
\hline Intrauterine device & 0.5 & $0.2-1.0$ & 0.4 & $0.1-1.4$ & 0.6 & $0.2-2.1$ \\
\hline Parity $>0$ & 1.3 & $0.7-2.6$ & 0.4 & $0.2-0.9$ & 0.8 & $0.3-2.3$ \\
\hline \multicolumn{7}{|l|}{$\mathrm{Age}^{\mathrm{c}}$} \\
\hline $\begin{array}{l}25-39 \text { years } \\
\geq 40 \text { years }\end{array}$ & $\begin{array}{l}0.6 \\
0.7\end{array}$ & $\begin{array}{l}0.2-1.6 \\
0.2-2.1\end{array}$ & $\begin{array}{l}0.6 \\
1.5\end{array}$ & $\begin{array}{l}0.2-1.8 \\
6.4-5.2\end{array}$ & $\begin{array}{l}0.8 \\
1.1\end{array}$ & $\begin{array}{l}0.2-3.6 \\
0.2-5.9\end{array}$ \\
\hline
\end{tabular}

a Number of women: irregular cycles $=448$, amenorrhea $=437$, cycles $>33 \mathrm{~d}=437$.

b Compared with nonsmoker or ex-smoker.

c Compared with age $<25$ years.

Microclimate. In general irregular cycles and amenorrhea were associated with cold temperatures, whereas long cycles were not. Temperature variability was associated with irregular cycles (table 5).

Other work conditions. No associations were shown between any cycle anomalies and the domestic and salaried work conditions studied (table 6), nor between cycle anomalies and variables such as those related to work speed or repetitive work (not shown).

The multiple logistic regression models for cycle anomalies are shown in table 7. In general the confidence intervals were wider for amenorrhea and long cycles than for irregular cycles, due to the smaller numbers. Several tendencies could be noted. Schedule and temperature variability were independently associated with cycle irregularity; these variables did not interact (data not shown). Cold temperatures were associated with amenorrhea, and schedule variability was related to long cycles.

\section{Discussion}

This analysis has shown that cycle anomalies such as irregular cycles, amenorrhea, and long cycles were related to characteristics of work, such as cold exposure and schedule variability, in poultry slaughterhouses. This analysis was conducted on a sample of 475 women after the exclusion of those taking oral contraceptives. With this sample size, the power of the study was reasonably good for work conditions with a high frequency. For example, 635 of the women worked on an assembly line; the power was thus 95 or $69 \%$, respectively, for detecting a twofold increase in the risk of irregular cycles or long cycles. On the average, the work conditions considered in this study concerned more than $30 \%$ of the women, and therefore a good probability of detecting differences in cycle regularity was ensured. On the other hand, results concerning rarer conditions, such as handling weights, should be regarded with caution.

\section{Possible bias}

Since data on occupational factors and menstrual cycle characteristics were collected retrospectively, we cannot entirely exclude the possibility of recall or selection bias. Selection bias was unlikely, however, since data were collected during the annual visit with the occupational health physician. This visit is mandatory, and there is no reason to suppose that women with menstrual disorders were examined more or less of ten than other women. Recall bias also appears to us to be improbable because neither the respondents nor the occupational health physicians should have been influenced by previous studies, since little information has been available on the effects of work conditions on the menstrual cycle.

\section{Prevalence of cycle irregularity}

The prevalence of cycle irregularity over the previous year in this sample was similar to that found (with no reference period given) among unselected populations of Australian women (19), although lower than that found among day workers in Japanese hospitals (11). We were unable to compare the prevalence of amenorrhea or long cycles by our definition, since the existing studies used much younger populations, such as college students, or used more extreme definitions of amenorrhea and long cycles $(15,16)$. In order to look at effects in small groups of workers with relatively similar conditions, we used subclinical definitions of amenorrhea and long cycles. 


\section{Schedule variability and cycle anomalies}

Variable schedules were associated with cycle anomalies in this population of menstruating slaughterhouse and cannery workers. This result is consistent with that obtained for hospital workers in Japan (11). An effect of schedule variability on reproduction is borne out by the association of irregular and inconvenient work schedules with adverse pregnancy outcome in a Swedish study of hospital workers (20).

It is interesting that work in alternating morning and afternoon 8-h shifts did not follow the pattern shown by less formal irregular work patterns. Nurminen (21) showed that shift workers had about a 1.5-fold risk of menstrual irregularity compared with nonshift workers. However, our study samples had important differences. Her sample was composed of workers from a variety of sectors of the economy, and onefourth of those assigned to multiple shifts were in manufacturing and related occupations. Our sample was homogeneous since all of the women were manual workers in slaughterhouses or canneries and none of them were assigned to night shifts. In addition the shiftworkers in our sample were not being compared with workers assigned to a regular, 8-h day, but with those working irregular hours. Women working two shifts had more regular hours of beginning and ending the workday than the women in nonshift work. Thus regular predictable schedule variations do not seem to affect hormonal cycling in the same way as irregular schedules.

\section{Cold temperatures}

Cold temperature was associated with irregular cycles and amenorrhea. Variations in temperature were associated with irregular cycles. It is interesting to speculate on the possibility that temperature changes may be involved in the control of both reproductive and circadian cycles. An association between cold and amenorrhea is supported by a study showing amenorrheic women to be less cold sensitive than eumenorrheic women because of hormonal interactions with the thermoregulatory system (22). Cold exposure in Quebec poultry slaughterhouses was recently shown to be associated with increased dysmenorrhea, another finding suggesting a relationship between cold and hormonal levels (23).

\section{Type of factory}

Being employed in a slaughterhouse (as opposed to a cannery) was associated with irregular cycles and with periods of amenorrhea. However, the slaughterhouses and canneries differed in too many ways for it to be appropriate to include factory type in the logistic regression. Irregular cycles were not associated with being uncomfortably cold within slaughterhouses, where $96 \%$ of the workers were exposed to tempera- tures of less than $18^{\circ} \mathrm{C}$. Irregular cycles were associated with schedule variability to an equal extent in the canneries and the slaughterhouses, although such schedules were much more prevalent among the slaughterhouse workers.

\section{Tobacco use}

Use of tobacco was associated with a twofold increase in the odds ratio for irregular cycles, long cycles, and amenorrhea, although the results for amenorrhea were significant after control for all other factors. Tobacco use has been related to early menopause and other reproductive problems $(17,24)$. In our study, the association between smoking and cycle anomalies was not totally mediated through low body weight, as has been suggested by others (25), since the association persisted after control for body weight.

\section{Biological indicators of female reproductive problems}

For male workers, sperm samples provide a readily available means of monitoring current reproductive health $(26-28)$. No noninvasive technique exists for sampling ova, and pregnancies are a relatively rare event, whose monitoring may be complicated by an "infertile worker effect" (29). It is possible that cycle anomalies could be a candidate for biological indicators of a potential for some reproductive problems of occupational origin, particularly those related to hormonal status. This study purposely chose indicators of low severity, at a level not necessarily pathological. Thus their prevalence was sufficiently high to permit associations to be found with occupational variables within a single workplace. It will be important to test the feasibility of applying methods which determine the regularity of the menstrual cycle and other parameters with somewhat more precision, such as personal calendars, to the workplace (30). These indicators may be useful in occupational health research in view of the difficulties of predicting health problems for small populations (31).

\section{Acknowledgments}

This study was made possible by collaboration between INSERM Unit 149, the Regional Labour Medical Inspections in Pays de Loire (Dr A Touranchet) and in Bretagne (Dr M Verger), the Institute of Occupational Health in Rennes (Professor M Curtès), and a team of occupational health physicians from both regions (Drs Bonneau, Bourgault, Brigadeau, Caillon, Cannec, Danielou, Dano, Georgelin, Hameau, Jublin, Kervennic, Le Breton, Lemercier, Lemeur, Leroux, Maupeu, Mauvieux, Mouret, Pilet, Romé, Saluden, Tossot, Touzard. Trehen) and their assistants. We 
thank the directors of the participating factories and the employees for their active contribution to the study.

Karen Messing was the recipient of a Quebec-France Collaborative Fellowship and a grant from the Institut de recherche en santé et en sécurité du travail du Québec. The study was partly funded by the Department of Labour of France (SES) and by the Regional Health Authorities (DRASS) in Bretagne and Pays de Loire.

\section{References}

1. Institut National de la Statistique et des Etudes Economiques (INSEE). Enquête sur l'emploi de 1986. INSEE, 1986:534. (Les Collections de l'INSEE; D114.)

2. Saurel-Cubizolles MJ, Bourgine $\mathbf{M}$, Touranchet A, Kaminski M. Enquête dans les abattoirs et les conserveries des régions Bretagne et Pays-de-Loire: conditions de travail et santé des salariés. Villejuif (France): Institut National de la Santé et de la Recherche Médicale, Unité 149, 1991. (Rapport à la Direction Régionale des Affaires Sanitaires et Sociales des Pays-de-Loire, Paris.)

3. Bue J, Cristofari MF. Contraintes et nuisances dans la vie de travail: 2ème série de rédultats de l'enquête nationale sur les conditions de travail, réalisée en mars 1984. Dos Stat Trav l'Emploi 1986;20:5-95.

4. Mergler D, Brabant C, Vézina N, Messing K. The weaker sex?: men in women's working conditions report similar health symptoms. J Occup Med 1987;29:417-21.

5. Savitz JA, Harlow SD. Selection of reproductive health endpoints for environmental risk assessment. Environ Health $1991 ; 90: 159-64$.

6. Office of Technology Assessment, United States Congress. Reproductive health hazards in the workplace. Washington, DC: US Government Printing Office, 1985. (Catalogue no OTA-BA-266.)

7. Mills JL, Jefferys J, Stolley PD. Effects of occupational exposure to estrogen and progestogens and how to detect them. J Occup Med 1984;26:269-72.

8. Harrington JM, Stein GF, Rivera RO, Morales AV. The occupational hazards of formulating oral contraceptives. Int Arch Environ Health 1978;1:12-5.

9. Panova $Z$. Menstrual and reproductive problems and gynaecological morbidity in women occupationally exposed to petrol. Leptose $\mathrm{Na}$ Hiegienno-Epidemiologicinata Sluzba 1976;20:53-6.

10. Zhou SY, Liang YX, Chen ZQ, Wang YL. Effects of occupational exposure to low-level carbon disulfide $\left(\mathrm{CS}_{2}\right)$ on menstruation and pregnancy. Ind Health 1988;26:203-14.

11. Uehata T, Sasakawan N. The fatigue and maternity disturbances of night workwomen. J Human Ergol 1982;11:465-74.

12. Toriola AL, Mathur DN. Menstrual dysfunction in Nigerian athletes. $\mathrm{Br} \mathbf{J}$ Obstet Gynaecol 1986;93: 979-85.

13. Frisch RE, Wyshak G, Vincent L. Delayed menarche and amenorrhea in ballet dancers. N Engl J Med 1980; 303:17-9.

14. Lemasters G, Hagen A, Samuels SJ. Reproductive outcomes in women exposed to solvents in 36 reinforced plastics companies: I. menstrual dysfunction. J Occup Med 1985;27:490-4.
15. Harlow SD, Matoski GM. The association between weight, physical activity and stress and variation in the length of the menstrual cycle. Am J Epidemiol 1991; 133:38-49.

16. McFalls JA. The risks of reproductive impairment in the later years of childbearing. Ann Rev Sociol 1990; $16: 491-519$.

17. Howe G, Westhoff C, Vessey M, Yeates D. Effects of age cigarette smoking and other factors on fertility: findings in a large prospective study. Br Med J 1985;290: 1697-700.

18. Tierney D, Romito P, Messing K. And she ate not the bread of idleness: exhaustion is related to domestic and salaried working conditions among Québec hospital workers. Women Health 1990;16:21-42.

19. Wood C, Larsen L, Williams R. Menstrual characteristics of 2343 women attending the Shepherd Foundation. Aust NZ J Obstet Gynaecol 1979;19:107-10.

20. Axelsson G, Rylander R, Molin I. Outcome of pregnancy in relation to irregular and inconvenient work schedules. Br J Ind Med 1989;46:393-98.

21. Nurminen T. Shift work, fetal development and course of pregnancy. Scand J Work Environ Health 1989;15: $395-403$.

22. Graham TE, Viswanathan N, Van Dijk JP, Bonen A, George JC. Thermal and metabolic responses to cold by men and by eumenorrheic and amenorrheic women. J Appl Physiol 1989;67:282-90.

23. Mergler D, Vézina N. Dysmenorrhea and cold exposure. J Reprod Med 1985;30:106-11.

24. Whelan EA, Sandler D, McConnaughey DR, Weinberg $C R$. Menstrual and reproductive characteristics and age at natural menopause. Am J Epidemiol 1990;131: $625-32$.

25. Sloss EM, Fredrichs RR. Smoking and menstrual disorders. Int J Epidemiol 1983;12:107-9.

26. Katz D, Andrew JB, Overstreet JL. Biological basis of in vitro tests of sperm function. In: Institute for Health Policy Analysis. Sperm measures and reproductive success. New York, NY: Alan R Liss, Inc, 1989:95-106.

27. Levine R. Methods for detecting occupational causes of male infertility: reproductive history versus semen analysis. Scand J Work Environ Health 1983;9:371-6.

28. Wyrobek A, Gordon LA, Burkhart JG, Francis MW, Kapp RW Jr, Letz G, et al. An evaluation of human sperm as indicators of chemically induced alterations of spermatogenic function: a report of the US Environmental Protection Agency Gene-Tox Program. Mutat Res 1983;115:73-148.

29. Joffe M. Biases in research on reproduction and women's work. Int J Epidemiol 1985;14:118-23.

30. Harlow S, Matanoski GM. The association between weight, physical activity, and stress and variation in the length of the menstrual cycle. Am J Epidemiol 1991; 133:38-49.

31. Messing K. Occupational health and safety of Canadian women. Ottawa (Canada): Ministry of Labour, 1991. (Catalogue no L016-1748/91E.)

Received for publication: 22 July 1991 